\title{
Review Article: Challenge in determining the crystal structure of epitaxial 0001 oriented $s p^{2}-B N$ films
}

Mikhail Chubarov, Hans Högberg, Anne Henry, and Henrik Pedersen

Citation: Journal of Vacuum Science \& Technology A 36, 030801 (2018); doi: 10.1116/1.5024314

View online: https://doi.org/10.1116/1.5024314

View Table of Contents: http://avs.scitation.org/toc/jva/36/3

Published by the American Vacuum Society

\section{Articles you may be interested in}

Review Article: Stress in thin films and coatings: Current status, challenges, and prospects Journal of Vacuum Science \& Technology A 36, 020801 (2018); 10.1116/1.5011790

High-temperature molecular beam epitaxy of hexagonal boron nitride layers

Journal of Vacuum Science \& Technology B, Nanotechnology and Microelectronics: Materials, Processing, Measurement, and Phenomena 36, $02 \mathrm{D} 103$ (2018); 10.1116/1.5011280

Predicting synergy in atomic layer etching

Journal of Vacuum Science \& Technology A: Vacuum, Surfaces, and Films 35, 05 C302 (2017);

10.1116/1.4979019

Etch considerations for directed self-assembly patterning using capacitively coupled plasma Journal of Vacuum Science \& Technology A: Vacuum, Surfaces, and Films 36, 031301 (2018); $10.1116 / 1.5004648$

Electronic structure of $\mathrm{BaSnO}_{3}$ investigated by high-energy-resolution electron energy-loss spectroscopy and ab initio calculations

Journal of Vacuum Science \& Technology A: Vacuum, Surfaces, and Films 36, 031503 (2018); $10.1116 / 1.5026298$

Band alignment at the $\mathrm{CdTe} / \mathrm{InSb}(001)$ heterointerface

Journal of Vacuum Science \& Technology A: Vacuum, Surfaces, and Films 36, 031101 (2018);

10.1116/1.5022799

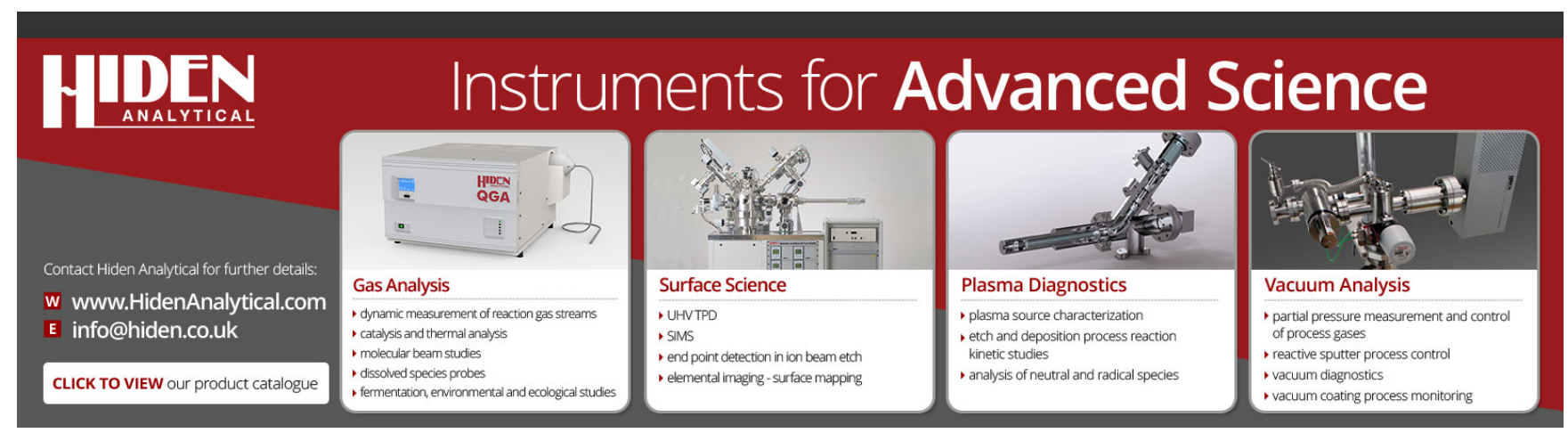




\title{
Review Article: Challenge in determining the crystal structure of epitaxial 0001 oriented $\mathbf{s p}^{2}-\mathrm{BN}$ films
}

\author{
Mikhail Chubarov, ${ }^{\text {a) }}$ Hans Högberg, Anne Henry, ${ }^{\text {b) }}$ and Henrik Pedersen ${ }^{\text {c) }}$ \\ Department of Physics, Chemistry and Biology, Linköping University, SE-581 83, Linköping, Sweden
}

(Received 31 January 2018; accepted 1 March 2018; published 20 March 2018)

\begin{abstract}
Boron nitride $(\mathrm{BN})$ as a thin film is promising for many future electronic applications. On 0001 $\alpha-\mathrm{Al}_{2} \mathrm{O}_{3}$ and $00014 \mathrm{H} / 6 \mathrm{H}-\mathrm{SiC}$ substrates, chemical vapor deposition yields epitaxial $\mathrm{sp}^{2}$-hybridized $\mathrm{BN}\left(\mathrm{sp}^{2}-\mathrm{BN}\right)$ films oriented around the c-axis. Here, the authors seek to point out that $\mathrm{sp}^{2}-\mathrm{BN}$ can form two different polytypes; hexagonal $\mathrm{BN}(\mathrm{h}-\mathrm{BN})$ and rhombohedral $\mathrm{BN}(\mathrm{r}-\mathrm{BN})$, only differing in the stacking of the basal planes but with the identical distance between the basal planes and in-plane lattice parameters. This makes structural identification challenging in c-axis oriented films. The authors suggest the use of a combination of high-resolution electron microscopy with careful sample preparation and thin film $\mathrm{x}$-ray diffraction techniques like pole figure measurements and glancing incidence (in-plane) diffraction to fully distinguish h-BN from r-BN. (c) 2018 Author(s). All article content, except where otherwise noted, is licensed under a Creative Commons Attribution (CC BY) license (http://creativecommons.org/licenses/by/4.0/). https://doi.org/10.1116/1.5024314
\end{abstract}

\section{INTRODUCTION}

Boron nitride (BN) as a thin film is promising for many future electronic applications. ${ }^{1-4}$ From chemical vapor deposition (CVD) and using triethylboron, $\mathrm{B}\left(\mathrm{C}_{2} \mathrm{H}_{5}\right)_{3}$, and ammonia, $\mathrm{NH}_{3}$, epitaxial growth of $\mathrm{sp}^{2}$-hybridized boron nitride $\left(\mathrm{sp}^{2}-\mathrm{BN}\right)$ has been carried out mainly on $0001 \alpha-\mathrm{Al}_{2} \mathrm{O}_{3}$ and $00014 \mathrm{H} / 6 \mathrm{H}-\mathrm{SiC}$ substrates, yielding films oriented around the c-axis. ${ }^{5-8}$ The processes developed for epitaxial growth requires temperatures in the range of $1100-1500^{\circ} \mathrm{C}$, thus motivating the choice of substrates. For a detailed account on CVD of $\mathrm{sp}^{2}-\mathrm{BN}$, the reader is referred to Ref. 9.

In particular for electronic applications, it is important to note that there are two different polytype of $\mathrm{sp}^{2}-\mathrm{BN}$ : hexagonal (h-BN) and rhombohedral (r-BN) (Fig. 1). ${ }^{9-12}$ h-BN exhibits an $\mathrm{ABAB}$... stacking sequence of the basal planes while an $\mathrm{ABCABC} .$. stacking sequence is observed in r-BN. In $\mathrm{h}-\mathrm{BN}$, each next basal plane is rotated $180^{\circ}$ around the c-axis ([0001]) with respect to the previous basal plane, while in $\mathrm{r}-\mathrm{BN}$, each next basal plane is shifted along the [1100] direction by $1.45 \AA$. It should be stressed that the only difference between h-BN and r-BN is the stacking sequence of the basal planes as the spacing between basal planes and in-plane lattice parameters are identical with 3.333 and $2.504 \AA$, respectively. ${ }^{13}$ The electronic properties of $\mathrm{h}-\mathrm{BN}$ and $\mathrm{r}-\mathrm{BN}$ differ (Table I) making structural characterization of $\mathrm{sp}^{2}-\mathrm{BN}$ films for electronic applications important.

In addition to $\mathrm{h}-\mathrm{BN}$ and $\mathrm{r}-\mathrm{BN}$, theoretical works predict other possible crystal structures for $\mathrm{sp}^{2}$-BN with a two layer

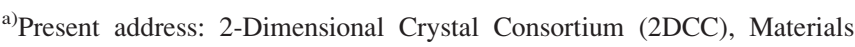
Research Institute, The Pennsylvania State University, University Park, PA 16802.

${ }^{b)}$ Deceased.

${ }^{c)}$ Electronic mail: henrik.pedersen@liu.se
}

stacking of $\mathrm{sp}^{2}$-BN basal planes with c-axis lattice constant of $6.66 \AA$ that are formed by rotating the B layer by different angles around c-axis or shifting it along different in-plane axis. ${ }^{17}$ These structures are closely related to h-BN. Structure factor calculations on such crystals using the VESTA software package ${ }^{18}$ show that these cannot be confused with r-BN if proper characterization tools are employed, while distinction between these structures and h-BN is more difficult due to the similarities in diffraction patterns and crystal structure variations that cannot be distinguished by electron microscopy due to the resolution limitations.

For a powder sample of polycrystalline $\mathrm{sp}^{2}-\mathrm{BN}$, determination of the crystal structure is straightforward from $\mathrm{x}$-ray diffraction (XRD) in $\theta-2 \theta$ geometry, ${ }^{12,19-21}$ whereas for epitaxial $\mathrm{sp}^{2}-\mathrm{BN}$ films, this distinction is more difficult due to the choice of substrates resulting in predominantly 0001 oriented films. Here, we seek to point to the impossibility to apply XRD in $\theta-2 \theta$ geometry, low magnification transmission electron microscopy (TEM), Raman and Fourier transform IR spectroscopy for structure determination of epitaxial c-axis oriented $\mathrm{sp}^{2}$-BN films. We suggest a combination of advanced thin film $\mathrm{x}$-ray diffraction techniques like pole figure measurements and glancing incidence (in-plane) (GI) diffraction and high-resolution electron microscopy (HREM) with careful sample preparation to distinguish h-BN from r-BN.

\section{LIMITATIONS OF COMMONLY USED TECHNIQUES FOR SP²-BN FILM STRUCTURE DETERMINATION}

The typically employed techniques for determination of crystalline structure are XRD and TEM which provide information about the crystallinity and crystal structure of a 


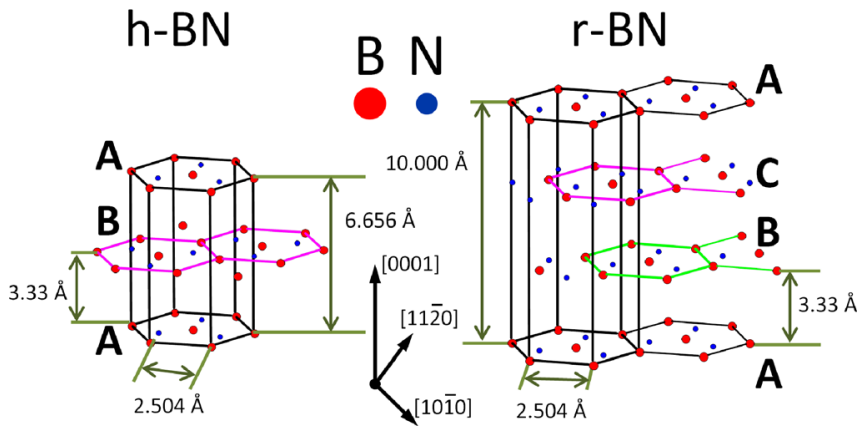

FIG. 1. (Color online) Hexagonal (left) and rhombohedral (right) crystal structures of $\mathrm{sp}^{2}-\mathrm{BN}$.

material. One would argue that Raman spectroscopy could be used for crystal structure determination as the technique is sensitive to the bonding and crystal symmetry. Due to the weak van der Waals interactions between the basal planes of $\mathrm{sp}^{2}-\mathrm{BN}$, the use of Raman spectroscopy is limited to $\mathrm{sp}^{3}-$ and $\mathrm{sp}^{2}-\mathrm{BN} .^{22,23}$

\section{A. X-ray diffraction}

In the literature, XRD in $\theta-2 \theta$ geometry is typically the technique used for structure determination of crystalline $\mathrm{sp}^{2}$ BN films. However, in such measurements, only the $000 \ell$ reflections will be detected as the $\mathrm{sp}^{2}$-BN films are predominantly grown along the c-axis. As h-BN and $\mathrm{r}-\mathrm{BN}$ have the same interplanar spacing, the $000 \ell$ reflections of h-BN and $\mathrm{r}$ $\mathrm{BN}$ will overlap making differentiation between the phases impossible. Figure 2 shows a typical XRD pattern from a $\mathrm{sp}^{2}$-BN thin film deposited on $\alpha-\mathrm{Al}_{2} \mathrm{O}_{3}$ with an AlN buffer layer. Peaks related to the diffraction from adjacent basal planes of $\mathrm{sp}^{2}-\mathrm{BN}$ and second order diffraction from these planes are marked by (0002) h-BN/(0003) r-BN and (0004) h-BN/(0006) r-BN, respectively. Shoulders at the lower $2 \theta$ angles of both peaks are visible and attributed to less ordered form of $\mathrm{sp}^{2}-\mathrm{BN}$ - turbostratic $\mathrm{BN}$ ( $\left.\mathrm{t}-\mathrm{BN}\right)$. Additionally, peaks from the $\alpha-\mathrm{Al}_{2} \mathrm{O}_{3}$ substrate and $\mathrm{AlN}$ buffer layer are visible and are marked correspondingly.

For more advanced XRD measurements of asymmetric planes, overlap between the reciprocal lattice points of the $\mathrm{sp}^{2}$-BN film and the substrate are likely to cause problems, which can be circumvented by employing high resolution instruments with monochromatic radiation. Lab scale instruments with high resolution optics and highly monochromatic $\mathrm{X}$-rays are characterized by low intensity. This will complicate measurements since boron and nitrogen are light elements with low electron density that will scatter $\mathrm{x}$-rays weakly.

TABLE I. Electronic properties of $h-$ and $r-B N$.

\begin{tabular}{lcc}
\hline \hline & h-BN & r-BN \\
\hline Bandgap (eV) & 5.955 indirect (Ref. 14), & 5.7 optical (Ref. 12), \\
& 5.971 direct (Ref. 10) & 3.9 indirect (ab initio \\
calculations) (Ref. 15) & Not reported \\
Electron effective mass & $0.26 \mathrm{~m}_{0}$ (Ref. 16) & Not reported \\
Hole effective mass & $0.47 \mathrm{~m}_{0}$ (Ref. 16) & \\
\hline \hline
\end{tabular}

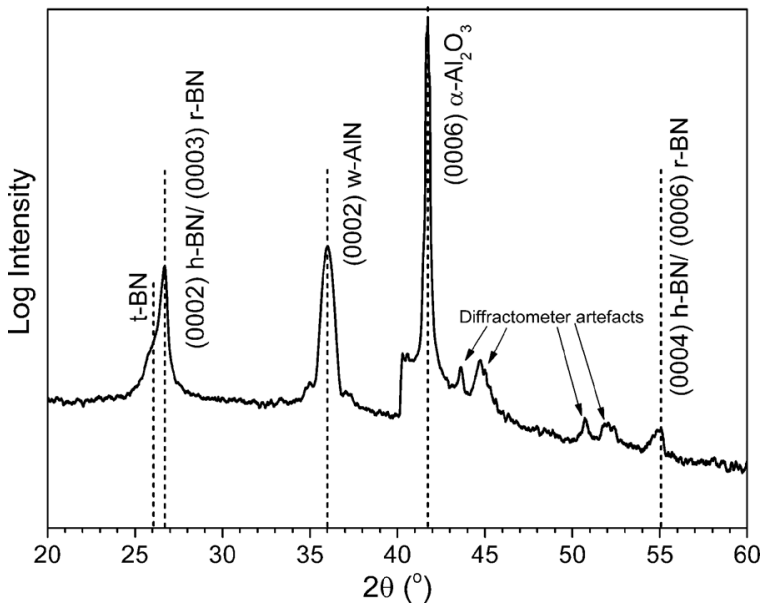

FIG. 2. $\theta-2 \theta$ XRD pattern from a crystalline $\mathrm{sp}^{2}$-BN thin film deposited on $\alpha-\mathrm{Al}_{2} \mathrm{O}_{3}$ with wurzitic $\mathrm{AlN}$ (w-AlN) buffer layer.

Consequently, asymmetric reflections, for instance in h-BN, will not be visible due to the low intensity of the diffracted beam comparable to the background level. A higher brilliance of the primary $\mathrm{x}$-ray beam, as in a synchrotron facility, will resolve the diffraction peak from the background level. ${ }^{24}$

\section{B. Transmission electron microscopy}

Conventional low resolution cross section TEM has frequently been used for observing the microstructure of deposited $\mathrm{sp}^{2}-\mathrm{BN}$ thin films in the literature. An example of a typical TEM micrograph is presented in Fig. 3 where basal planes of the $\mathrm{sp}^{2}-\mathrm{BN}$ are discernable. As can be seen, it is only possible to observe that the distance between basal planes is around $3.35 \AA$. This is not conclusive for the determination of the crystalline structure as the distance between the basal planes is identical for h-BN and r-BN.

\section{DEMANDS FOR STRUCTURE DETERMINATION OF SP ${ }^{2}$-BN FILMS}

\section{A. X-ray diffraction}

For $\theta-2 \theta$ XRD, one could argue that due to different structure factors, it should be possible to differentiate between $\mathrm{h}-\mathrm{BN}$ and $\mathrm{r}-\mathrm{BN}$ by comparing the relative intensities of the diffraction from the adjacent basal planes and second order diffraction from them, i.e., the 0002 and 0004 for h-BN and 0003 and 0006 for r-BN. In case of h-BN and

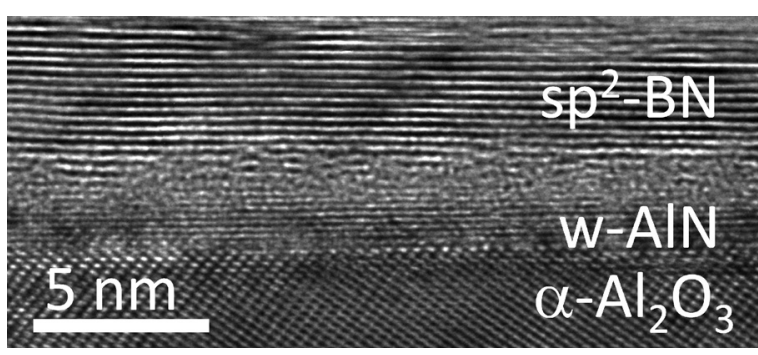

FIG. 3. Cross section TEM image of $\mathrm{sp}^{2}-\mathrm{BN}$ deposited on $\alpha-\mathrm{Al}_{2} \mathrm{O}_{3}$ with w-AlN buffer layer. The amorphous inclusion in between the AlN buffer layer and $\mathrm{sp}^{2}-\mathrm{BN}$ film is an effect of the TEM sample preparation. 
TABLE II. Powder diffraction files of h-BN and r-BN listing planes causing $\mathrm{Cu} \mathrm{K} \alpha$ diffraction peaks at $2 \theta$ angles with corresponding relative intensity (Ref. 13)

\begin{tabular}{lcccc}
\hline \hline $\begin{array}{l}\text { h-BN diffraction } \\
\text { peak }\end{array}$ & $\begin{array}{c}\text { r-BN diffraction } \\
\text { peak }\end{array}$ & $\begin{array}{c}\text { Position } \\
2 \theta(\mathrm{deg})\end{array}$ & $\begin{array}{c}\text { h-BN relative } \\
\text { intensity }\end{array}$ & $\begin{array}{r}\text { r-BN relative } \\
\text { intensity }\end{array}$ \\
\hline 0002 & 0003 & $26.76 / 26.72$ & 100 & 100 \\
$10 \overline{1} 0$ & - & 41.60 & 15 & - \\
$\overline{-}$ & $10 \overline{1} 1$ & 42.62 & - & 13 \\
$10 \overline{1} 1$ & - & 43.87 & 6 & - \\
$\overline{-}$ & $01 \overline{1} \overline{1}$ & 45.57 & - & 7 \\
$01 \overline{1} 2$ & - & 50.15 & 9 & - \\
0004 & 0006 & $55.16 / 55.06$ & 6 & 4 \\
- & $10 \overline{1} 4$ & 56.07 & - & 2 \\
\hline \hline
\end{tabular}

r-BN the ratio between these diffraction peaks intensities should be 100 to 6 and 100 to 4, respectively, according to Table II. Such differences are too small for a reliable structure determination.

Instead, thin film diffraction techniques such as pole figure measurements and glancing incidence diffraction of asymmetric peaks with $2 \theta$ angles unique for each structure are required to determine the symmetry of the structure, i.e., hexagonal or rhombohedral, bearing in mind potential overlap from substrate diffraction peaks in close vicinity in both $2 \theta$ and inclination angle which can hide the film peak. As listed in Table II, two such asymmetrical peaks are $10 \overline{1} 1$ and $01 \overline{1} 2$, where the different plane spacing make these pair of peaks appear at different $2 \theta$ angles and difference in c-axis length of two structures induce different inclination angles in pole figure measurements (angle between the plane under investigation and basal plane).

Furthermore from Table II, the $10 \overline{1} 0$ peak is unique for h$\mathrm{BN}$ suggesting that this peak could be monitored in pole figure measurements and serve as fingerprint for h-BN, albeit, for 0001 oriented h-BN films the $\psi$ angles between $\{10 \overline{1} 0\}$ and $\{0001\}$ is $90^{\circ}$. Since XRD pole figure measurements are usually limited to $85^{\circ} \psi$ angles, it is not possible to observe these set of planes. This means that GI-XRD (in-plane) must be performed as such geometry allow the observation of in

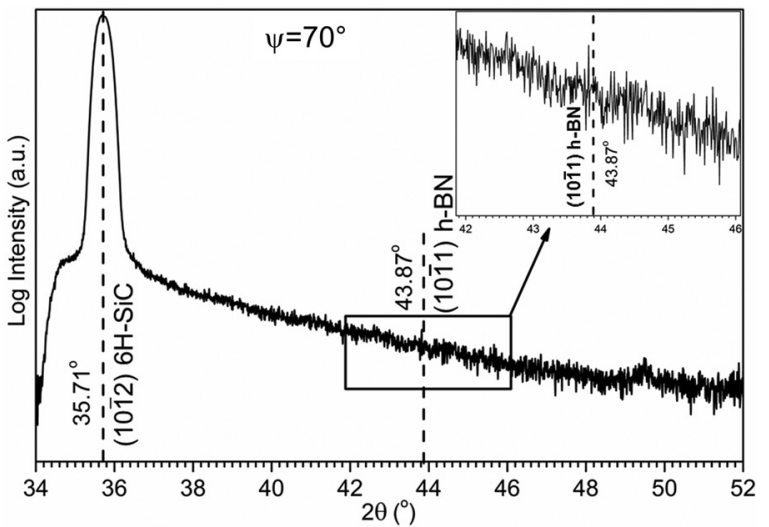

FIG. 5. $2 \theta$ scan around $10 \overline{1} 1$ diffraction peak of h-BN to establish if the pole at $70^{\circ}$ inclination in pole figure of h-BN originate from h-BN or is an effect of diffraction of nonmonochromatic x-ray radiation on (1012) planes of $6 \mathrm{H}-$ $\mathrm{SiC}$. Adapted with permission from Chubarov et al., Chem. Mater. 27, 1640 (2015). Copyright 2015, American Chemical Society.

plane ordering in the films under investigation and thus by observing or not observing (1010) planes of h-BN conclude if h- or $\mathrm{r}-\mathrm{BN}$ is formed.

Figure 4 shows pole figure measurements of the $10 \overline{1} 1$ poles of (a) $\mathrm{r}-\mathrm{BN}$ conducted at the corresponding diffraction angle $2 \theta=42.62^{\circ}$ (Ref. 25) and (b) h-BN at its diffraction angle $2 \theta=43.87^{\circ}$. This figure shows double peaks separated in $\phi$ (rotation) by $60^{\circ}$ and are about $70^{\circ}$ in $\psi$ (inclination). The set of six peaks observed at the inclination angle of around $70^{\circ}$ (marked with arrows from the inside of the pattern) is corresponding to the $6 \mathrm{H}-\mathrm{SiC}\{10 \overline{1} 2\}$ planes that exhibit an angle of $70^{\circ}$ to the $6 \mathrm{H}-\mathrm{SiC}\{0001\}$ planes. The second set of 6 peaks observed at $\psi$ of about $77^{\circ}$ (marked with arrows from outside of the pattern) corresponds to r-BN $\{10 \overline{1} 1\}$ planes that exhibit $77^{\circ}$ angle to the $\{0001\}$ planes of $\mathrm{r}-\mathrm{BN}$. Another conclusion is that $\mathrm{r}-\mathrm{BN}$ forms twinned crystals, since from the crystal structure this plane will show threefold symmetry with three peaks separated $120^{\circ}$ in $\phi$ angle. Thus, the film grows epitaxial to the $6 \mathrm{H}-\mathrm{SiC}$ substrate with two crystals $60^{\circ}$ rotated around the c-axis. For the $10 \overline{1} 1$ pole recorded from an h-BN film, the peaks appear at an
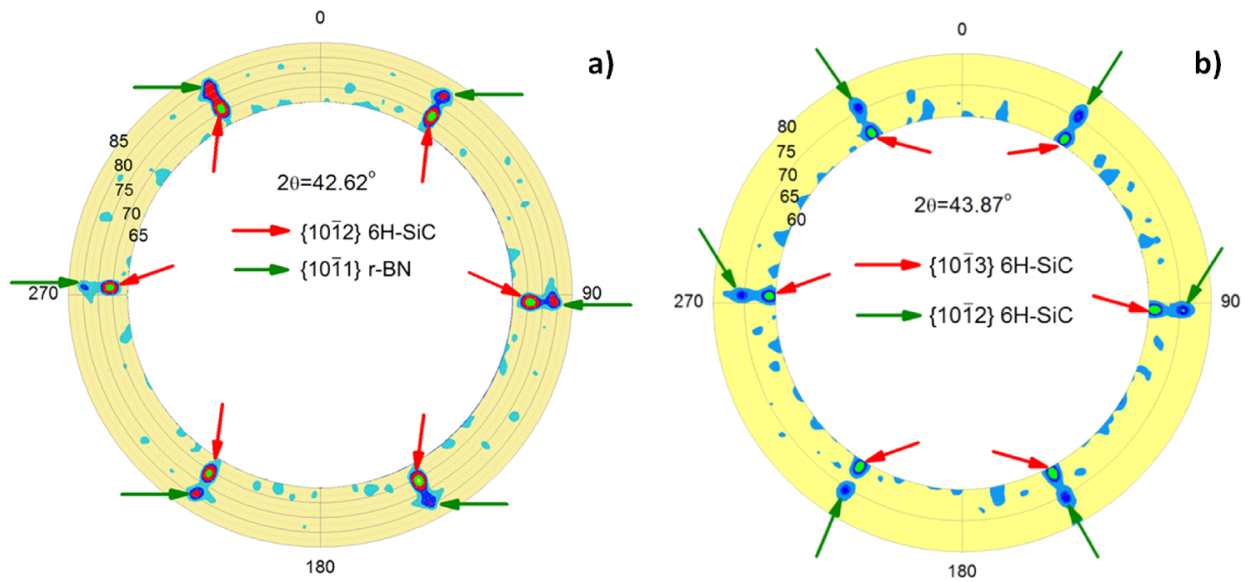

FIG. 4. (Color online) Pole figures recorded from the $\mathrm{sp}^{2}-\mathrm{BN}$ sample deposited on $6 \mathrm{H}-\mathrm{SiC}$ (a) at diffraction angle $42.62^{\circ}$ corresponding to spacing between (1011) planes of r-BN and (b) at diffraction angle of $43.87^{\circ}$ that corresponds to diffraction from (1011) planes of h-BN. Adapted with permission from Chubarov et al., Chem. Mater. 27, 1640 (2015). Copyright 2015, American Chemical Society. 
a

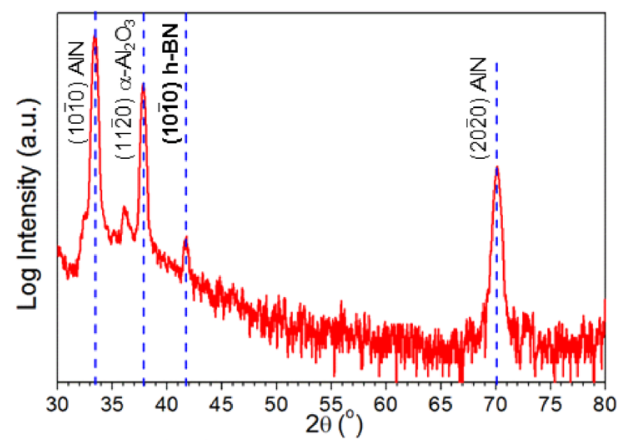

b

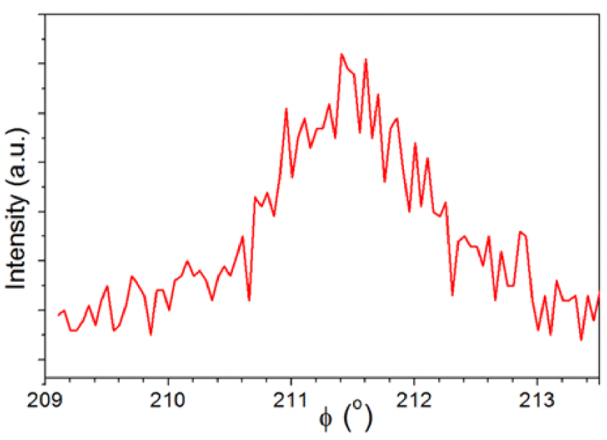

FIG. 6. (Color online) (a) 20 measurement performed in GI-XRD geometry showing presence of h-BN in the film and (b) $\phi$ scan at diffraction angle corresponding to $10 \overline{1} 0$ peak of h-BN. Reprinted with permission from Chubarov et al., Chem. Mater. 27, 1640 (2015). Copyright 2015, American Chemical Society.

appropriate $\psi$ of $70^{\circ}$ and could be interpreted as a proof for the presence of h-BN in the film. As can be concluded from Fig. 5 the peak at the $\psi$ of $70^{\circ}$ originates from the long tail of the diffraction peak of $10 \overline{1} 2$ of $6 \mathrm{H}-\mathrm{SiC}$ at a lower diffraction angle that has an intensity above the background level at higher diffraction angles due to the use of nonmonochromatic optics. If the measurement is done without taking such overlaps into account, resulting pole figure might be misinterpreted leading to the conclusion that h-BN is formed while it is not the case. Similar $2 \theta$ measurement was conducted for the case of r-BN and corresponding peak was clearly observed leaving no doubts about the conclusion.

Another way to observe the formation of h-BN is XRD measurements in glancing incidence geometry as is discussed before. Figure 6(a) shows $2 \theta$ measurement conducted in glancing incidence geometry that visualize diffraction peaks from $(10 \overline{1} 0)$ and $(20 \overline{2} 0)$ planes of $\mathrm{AlN},(11 \overline{2} 0)$ of $\alpha-\mathrm{Al}_{2} \mathrm{O}_{3}$ and (1010) of h-BN. Figure 6(b) represents $\phi$ scan at a diffraction angle that corresponds to $10 \overline{1} 0$ peak of h-BN. These figures confirm the formation of h-BN in the film, represent epitaxial relation and prove epitaxy itself by observing peaks that are present from the substrate and buffer. This is further confirmed by the observation that the h-BN peak is confined in $\phi$.

\section{B. High resolution electron microscopy}

From the atomic resolution available in modern highresolution electron microscopes it is possible following careful sample preparation to observe differences in stacking sequences as in h-BN and r-BN. Thus, the sample must be prepared in a way so that the incident electron beam is orthogonal to a crystal plane where it is possible to make a distinction between crystal structures. This approach requires either previous knowledge on the in-plane orientation of the $\mathrm{sp}^{2}-\mathrm{BN}$ crystal, or assumption on the epitaxial relation with the substrate. Figure 7 represents the crystal

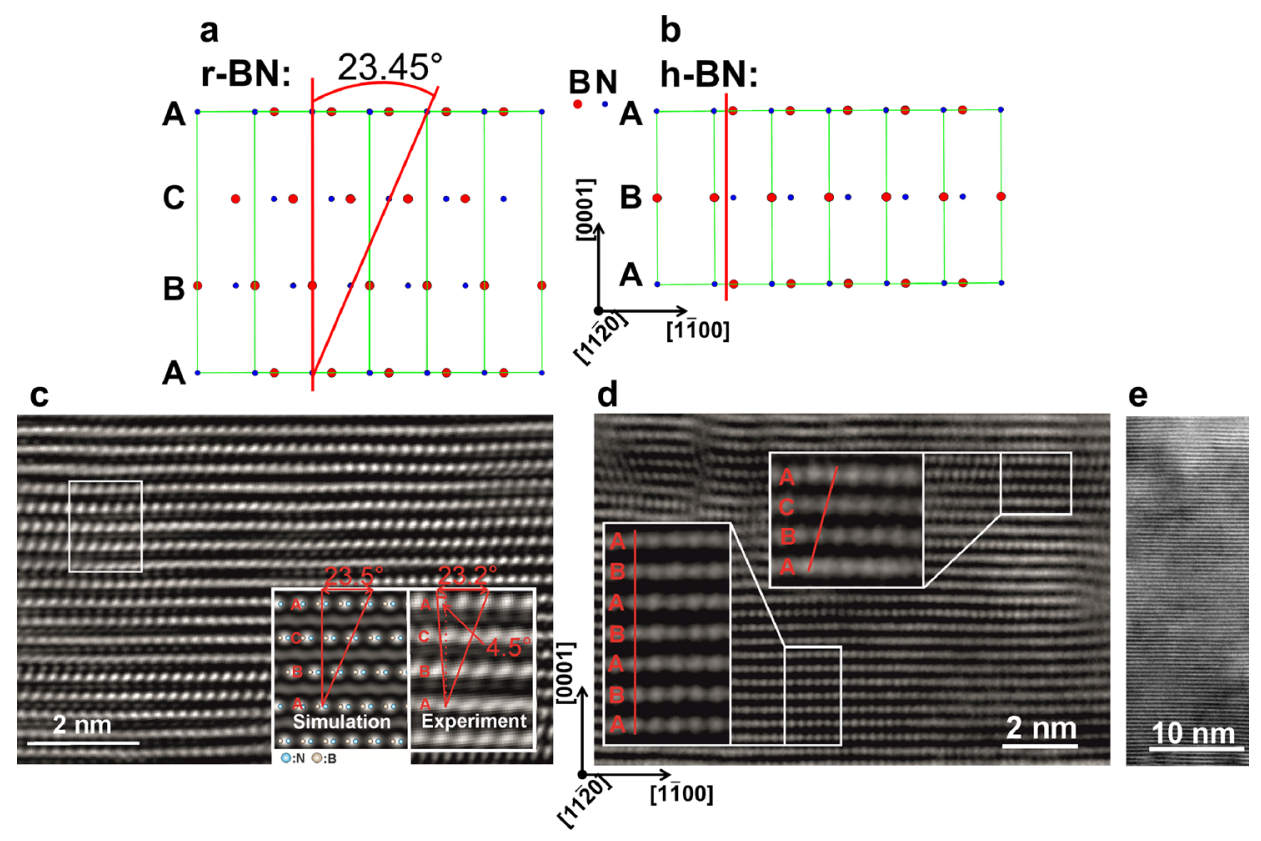

FIG. 7. (Color online) Drawing of the crystal structure of (a) r-BN and (b) h-BN exposing (1120) planes and TEM images of appropriately prepared sp ${ }^{2}$-BN samples. (c) $\mathrm{sp}^{2}-\mathrm{BN}$ on $6 \mathrm{H}-\mathrm{SiC}$ and (d) $\mathrm{sp}^{2}-\mathrm{BN}$ on $\alpha-\mathrm{Al}_{2} \mathrm{O}_{3}$ with AlN buffer layer. (e) shows low magnification TEM image of bulk sp ${ }^{2}$-BN. Reprinted with permission from Chubarov et al., Chem. Mater. 27, 1640 (2015). Copyright 2015, American Chemical Society. 
structures of (a) r-BN and (b) h-BN when the view plane is parallel with $(11 \overline{2} 0)$ planes of both crystals. In this case, the difference between $\mathrm{h}$ - and $\mathrm{r}-\mathrm{BN}$ is clearly visible where the $\mathrm{B}-\mathrm{N}$ atomic planes are following a line that has an angle of $23.45^{\circ}$ with $\mathrm{c}$-axis in $\mathrm{r}-\mathrm{BN}$ and where $\mathrm{B}-\mathrm{N}$ atomic pairs are following c-axis [lines are marked with red in Figs. 7(a) and 7(b)]. Figures 7(c) and 7(d) are accordingly prepared samples of $\mathrm{sp}^{2}-\mathrm{BN}$ deposited on $6 \mathrm{H}-\mathrm{SiC}$ and $\alpha-\mathrm{Al}_{2} \mathrm{O}_{3}$ substrate with AIN buffer layer, respectively. These images allow observing $\mathrm{r}$ - and h-BN formation in both layers where $\mathrm{r}-\mathrm{BN}$ is found in both samples while h-BN is suggested to be promoted for a limited thickness by the underlying AlN buffer layer. ${ }^{25}$ This approach is similar to that conducted by Sutter et al. to observe crystal structure of $\mathrm{sp}^{2}-\mathrm{BN}$ and reveal influence of the underlying material. ${ }^{11}$ Figure $7(\mathrm{e})$ represents low magnification TEM image of the $\mathrm{sp}^{2}-\mathrm{BN}$. TEM with electron diffraction (ED) are suitable tools for the determination of the crystalline structure of $\mathrm{sp}^{2}-\mathrm{BN}$ but care should be taken when analyzing data to avoid misinterpretation where, for example, in TEM other planes are observed or in ED from the substrate being attributed to the film. As always when using TEM, there is a risk that the observed area is not representative of the entire sample but is just a minor inclusion in the film that happened to be present in the prepared sample.

\section{CONCLUSION}

We argue that from a combination of advanced thin film $\mathrm{X}$-ray diffraction techniques like pole figure measurements and glancing incidence (in-plane) diffraction and HREM with careful sample preparation, it is possible to distinguish $\mathrm{h}-\mathrm{BN}$ from $\mathrm{r}-\mathrm{BN}$ in epitaxial c-axis oriented $\mathrm{sp}^{2}-\mathrm{BN}$ films. We see this as important to advance $\mathrm{BN}$ as an electronic material. Finally, we hope that this paper will encourage researchers to conduct more careful characterization of the material synthesized, allowing reported material properties and synthesis conditions being assigned to the correct $\mathrm{BN}$ polytype.

\section{ACKNOWLEDGMENTS}

M.C., H.P., and H.H. express their deepest appreciation to the late Anne Henry for giving them the chance to work with her in the field of BN CVD in general and for discussions to this work. Her passion and devotion to science will forever serve as a guiding light. This work was supported by the Swedish Research Council (VR: Grant No. 621-2013-5585), Carl Tryggers Stiftelse (No. 12:175) and the Swedish Foundation for Strategic Research (No. SSF IS-14-0024). H.H. acknowledges the Swedish Government Strategic Research Area in Materials Science on Functional Materials at Linköping University (Faculty Grant No. SFO-Mat-LiU 2009-00971) for financial support.

${ }^{1}$ H. X. Jiang and J. Y. Lin, Semicond. Sci. Technol. 29, 084003 (2014).

${ }^{2}$ S. Majety, J. Li, W. P. Zhao, B. Huang, S. H. Wei, J. Y. Lin, and H. X. Jiang, Appl. Phys. Lett. 102, 213505 (2013).

${ }^{3}$ N. Izyumskaya, D. O. Demchenko, S. Das, Ü. Özgür, V. Avrutin, and H. Morkoç, Adv. Electron. Mater. 3, 1600485 (2017).

${ }^{4}$ J. Bao, K. Jeppson, M. Edwards, Y. Fu, L. Ye, X. Lu, and J. Liu, Electron. Mater. Lett. 12, 1 (2016).

${ }^{5}$ Y. Kobayashi and T. Akasaka, J. Cryst. Growth 310, 5044 (2008).

${ }^{6}$ M. Chubarov, H. Pedersen, H. Högberg, Zs. Czigany, and A. Henry, CrystEngComm 16, 5430 (2014).

${ }^{7}$ Q. Paduano, M. Snure, D. Weyburne, A. Kiefer, G. Siegel, and J. Hu, J. Cryst. Growth 449, 148 (2016).

${ }^{8}$ A. Rice, A. Allerman, M. Crawford, T. Beechem, T. Ohta, C. Spataru, J. Fiegel, and M. Smith, J. Cryst. Growth 485, 90 (2018).

${ }^{9}$ M. Chubarov, H. Pedersen, H. Högberg, J. Jensen, and A. Henry, Cryst. Growth Des. 12, 3215 (2012).

${ }^{10}$ K. Watanabe, T. Taniguchi, and H. Kanda, Nat. Mater. 3, 404 (2004).

${ }^{11}$ P. Sutter, J. Lahiri, P. Zahl, B. Wang, and E. Sutter, Nano Lett. 13, 276 (2013).

${ }^{12}$ L. Xu, J. Zhan, J. Hu, Y. Bando, X. Yuan, T. Sekiguchi, M. Mitome, and D. Golberg, Adv. Mater. 19, 2141 (2007).

${ }^{13}$ Joint Committee on Powder Diffraction Standards, JCPDS, Swarthmore, PA, pattern $34-0421$; pattern $45-1171$.

${ }^{14}$ G. Cassabois, P. Valvin, and B. Gil, Nat. Photonics 10, 262 (2016).

${ }^{15}$ J. Furthmüller, J. Hafner, and G. Kresse, Phys. Rev. B 50, 15606 (1994).

${ }^{16}$ Y.-N. Xu and W. Y. Ching, Phys. Rev. B 44, 7787 (1991).

${ }^{17}$ N. Ooi, A. Rairak, L. Lindsley, and J. B. Adams, J. Phys.: Condens. Mater 18, 97 (2006).

18“"VESTA," http://jp-minerals.org/vesta/en/.

${ }^{19}$ Y. Kubota, K. Watanabe, O. Tsuda, and T. Taniguchi, Science 317, 932 (2007).

${ }^{20}$ K. Bao, F. Yu, L. Shi, S. Liu, X. Hu, J. Cao, and Y. Qian, J. Solid State Chem. 182, 925 (2009).

${ }^{21}$ L. Wang, R. Hang, Y. Xu, C. Guo, and Y. Qian, RSC Adv. 4, 14233 (2014).

${ }^{22}$ J. Liu, Y. K. Vohra, J. T. Tarvin, and S. S. Vagarali, Phys. Rev. B 51, 8591 (1995).

${ }^{23}$ S. Reich, A. C. Ferrari, R. Arenal, A. Loiseau, I. Bello, and J. Robertson, Phys. Rev. B 71, 205201 (2005).

${ }^{24}$ X-ray data booklet, Lawrence Berkley National Laboratory, LBNL/ PUB-490 Rev. 3, October 2009, http://xdb.lbl.gov/xdb-new.pdf.

${ }^{25}$ M. Chubarov, H. Pedersen, H. Hogberg, Zs. Czigany, M. Garbrecht, and A. Henry, Chem. Mater. 27, 1640 (2015). 